\title{
Laparoscopic sleeve gastrectomy versus endoscopic sleeve gastroplasty: a systematic review and meta-analysis
}

\section{다)(우우}

\author{
Authors \\ Giuseppe Marincola*, ${ }^{*}$, Camilla Gallo*,2,3, Cesare Hassan ${ }^{4}$, Luca Sessa ${ }^{1}$, Marco Raffaelli ${ }^{1}$, Guido Costamagna ${ }^{2,3}$, \\ Vincenzo Bove ${ }^{2,3}$, Valerio Pontecorvi ${ }^{2,3}$, Beatrice Orlandini ${ }^{2,3}$, Ivo Boškoski ${ }^{2,3}$
}

Institutions

1 Bariatric and Metabolic Surgery Unit, Fondazione Policlinico Universitario Agostino Gemelli IRCCS, Rome, Italy

2 Digestive Endoscopy Unit, Fondazione Policlinico Universitario Agostino Gemelli IRCCS, Rome, Italy

3 Centre for Endoscopic Research Therapeutics and Training (CERTT), Catholic University of Rome, Italy

4 Digestive Endoscopy Unit, Nuovo Regina Margherita, Rome, Italy

submitted 26.5.2020

accepted after revision 5.10 .2020

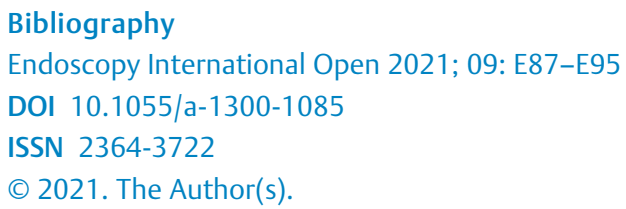

This is an open access article published by Thieme under the terms of the Creative Commons Attribution-NonDerivative-NonCommercial License, permitting copying and reproduction so long as the original work is given appropriate credit. Contents may not be used for commecial purposes, or adapted, remixed, transformed or built upon. (https://creativecommons.org/licenses/by-nc-nd/4.0/)

\section{Corresponding author}

Ivo Boškoski MD, PhD, Digestive Endoscopy Unit, Fondazione Policlinico Universitario Agostino Gemelli IRCCS, Rome, Italy, Centre for Endoscopic Research Therapeutics and Training (CERTT), Catholic University of Rome, Italy, Largo A. Gemelli, 8, 00168 Rome, Italy Fax: +390630156581

ivo.boskoski@policlinicogmelli.it

\section{ABSTRACT}

Background and study aims Laparoscopic sleeve gastrectomy (LSG) is the current standard for bariatric surgery, but it is affected by several postoperative complications. Endoscopic sleeve gastroplasty (ESG) was created as a less invasive alternative to LSG. However, its efficacy and safety compared with LSG is unclear.

Materials and methods Relevant publications were identified in MEDLINE/Cochrane/EMBASE/OVID/ PROSPERO and $\mathrm{NIH}$ up to January 2020. Studies were selected that included obese patients with a baseline body mass index (BMI) between 30 and $40 \mathrm{~kg} / \mathrm{m}^{2}$ with a minimum of 12 months of follow-up and with reported incidence of complications. The mean difference in percentage of excess weight loss (\%EWL) at 12 months between LSG and ESG represented the primary endpoint. We also assessed the difference in pooled rate of adverse events. The quality of the studies and heterogeneity among them was analyzed.

Results Sixteen studies were selected for a total of 2188 patients (LSG: 1429; ESG: 759) with a mean BMI 34.34 and $34.72 \mathrm{~kg} / \mathrm{m}^{2}$ for LSG and ESG, respectively. Mean \%EWL was $80.32 \%\left( \pm 12.20 ; 95 \% \mathrm{Cl} ; P=0.001 ; I^{2}=98.88\right)$ and $62.20 \%$ $\left( \pm 4.38 ; 95 \% \mathrm{Cl} ; P=0.005 ; I^{2}=65.52\right)$ for the LSG and ESG groups, respectively, corresponding to an absolute difference of $18.12 \%( \pm 0.89 ; 95 \% \mathrm{Cl}, P=0.0001)$. The difference in terms of mean rate of adverse events was $0.19 \%( \pm 0.37$; $\left.95 \% \mathrm{Cl} ; X^{2}=1.602 ; P=0.2056\right)$.

Conclusions Our analysis showed a moderate superiority of LSG versus ESG. No difference in terms of safety was shown between the two groups. ESG is a less-invasive, repeatable and reversable and acceptable option for mildmoderate obese patients.

\section{Introduction}

Obesity - defined as body mass index (BMI) $\geq 30 \mathrm{~kg} / \mathrm{m}^{2}$ - is a chronic and multifactorial condition characterized by abnormal weight gain due to excessive adipose tissue accumulation that represents a worldwide growing challenge for public health. In Europe, a total of $€ 81$ billion has been estimated to be spent per

\footnotetext{
* Co-shared authorship
}

year for bariatric patients' management [1].

Obesity requires a multidisciplinary approach to both prevention and treatment. Surgery has been demonstrated to be the most effective treatment for severe obesity in terms of long-term weight loss, comorbidities, and quality of life (QoL) improvements and overall mortality decrease [2]. Laparoscopic sleeve gastrectomy (LSG) represents the most common procedure, accounting for $59.4 \%$ of the 228,000 annual bariatric sur- 
gical procedures performed in the United States [3]. LSG is resection of the gastric greater curvature and of the fundus of the stomach through a partial vertical gastrectomy, which leads to a gastric tubulization. The final result is effective body weight loss and improvement in QoL and comorbidities. However, LSG is associated with acute and chronic postoperative complications, such as bleeding, leakage, and gastric fistulae [4], which sometimes discourage patients and make it less desirable to them.

The recent emergence of bariatric endoscopic techniques promises less invasive, more cost-effective, and repeatable [5] approaches to the treatment of obesity. Endoscopic sleeve gastroplasty (ESG) consists of tubularization of the gastric cavity created by placing full-thickness sutures in a triangle and in a top-to-bottom direction from the gastric angulus towards the gastric fundus, preserving the area of the pyloric antrum and a part of the fundus itself. This procedure limits the amount of food that can be introduced into the stomach and reduces the number of calories consumed, so it was thought to potentially achieve the same results obtained through surgery. Scientific evidence related to the outcomes of ESG is still limited, but according to the latest studies, this endoscopic procedure is associated with a low rate of adverse events (AEs) (1.1\%) [6].

ESG was created as a less invasive and more cost-effective endoscopic alternative to LSG, but very few comparative studies are available. Therefore, a proper meta-analysis that combines the data from the two techniques is an unmet need.

The primary aim of this systematic review was to compare the efficacy and safety of the surgical and endoscopic bariatric approaches to understand if these two interventions are interchangeable for the same obese population.

\section{Materials and methods}

\section{Patients and PICO model}

The meta-analysis was planned according to PICO format: the P-population included samples of patients with a mean baseline BMI between 30 and $40 \mathrm{~kg} / \mathrm{m}^{2}$ and with a minimum follow-up time of 12 months after the endoscopic or surgical intervention; LSG was considered as the I-intervention performed on the population involved and ESG was identified as the C-comparison; the main $\mathrm{O}$-outcome evaluated was the difference between the procedures in terms of efficacy (expressed by the percentage of Excess of Weight Loss (\%EWL) at 12 months); the secondary $\mathrm{O}$-outcome was the difference between the procedures in terms of safety (expressed by the rate of peri-procedural major and/or minor adverse events).

\section{Research methods and articles inclusion criteria}

The Cochrane Highly Sensitive Search Strategy [7] was used to conduct a systematic literature research about studies published till January 2020 on MEDLINE [Pubmed], OVID, Cochrane CENTRAL, EMBASE, PROSPERO and NIH. Other researches have been performed on ClinicalTrials.gov, World Health Organization (WHO) and International Clinical Trials Registry Platform (ICTRP). Additional information was retrieved through a Google research and a Gray literature research was performed. The ar- ticles selection was conducted using the MeSH terms: "Weight loss", "Obesity", "Bariatric", "Gastroplasty", "Overstitch", "Endoscopic sleeve gastroplasty”, "ESG”, "Bariatric endoscopy”, "Endobariatrics”, "Bariatric surgery”, "Laparoscopic sleeve gastrectomy”, “LSG”, “SG”, "Sleeve”, “Gastrectomy”; these key words were used in all possible combinations to collect the maximum number of articles. Among all articles identified, only published articles about ESG or LSG on humans, written in English, with full text based on their title and abstract were included. Non-human studies, unpublished studies, experimental studies in animal models, single case reports, technical reports, reviews, abstracts, editorials and studies in other languages than English were not included. Studies conducted on groups of patients with a mean baseline $\mathrm{BMI}<30 \mathrm{~kg} / \mathrm{m}^{2}$ or $>40 \mathrm{~kg} / \mathrm{m}^{2}$ were excluded, as well as studies with $<12$ months follow-up and/or not reporting the rate of peri-procedural AEs.

This systematic review and meta-analysis was performed in accordance with the Preferred Reporting Items for Systematic Review and Meta-analysis (PRISMA) statement [8] and Cochrane Handbook for Systematic Reviews of Intervention [9]. Eligibility criteria were determined by two authors (G.M. and C.G.), who independently evaluated the studies.

\section{Data collection and statistical analysis}

All data identified were downloaded and combined into a reference manager database (EndNote X9) and any duplicate citation identified was removed. A standardized data extraction form was developed based on the Data Extraction and Assessment Template from the The Cochrane Public Health Group [10]. For some studies, data were extracted indirectly using WebPlotDigitizer version 3.10. Two authors (G.M. and C.G.) independently collected all relevant data in spreadsheets: study design; total study duration; inclusion and exclusion criteria; age, sex, mean baseline BMI and total number of participants; type of intervention and device used; mean 12 months \%EWL; number and type of peri-procedural AEs. Any discrepancies were resolved by group discussion.

The meta-analysis was performed by computing continuous and dichotomous outcomes for each study. Mean percent of excess weight loss (\%EWL) at 12 months after LSG or ESG and standard deviations (SD) with $95 \%$ confidence intervals (Cls) pooled and used as weighted means (WM) in Der Simonian and Laird random effects model for both groups [11]. Weighted mean difference (WMD) was used between the two pooled means; pooled standard deviations with $95 \% \mathrm{Cl}$ and $P$ values were calculated [12]. Peri-procedural AE rate was pooled using random effects model, and comparison of proportions was computed using chi-squared test $[13,14]$ and the mean difference between the two arms was computed as above. Both major and minor AEs were considered, in accordance with ClavienDindo classification [15]

To evaluate the quality of the studies included, the Revised Cochrane Risk-Of-Bias (ROB) tool for randomized trials [16] was applied to randomized studies. The Cochrane ROB combines information in five bias domains (bias arising from the randomization process, bias due to deviations from the intended interventions, missing outcome data, bias in measurement 
of the outcome and bias in selection of the reported result), to divide trials into categories of "low" or "high" quality studies. The NIH Quality Assessment Tool for Before-After (Pre-Post) Studies with No Control Group [17] was used to evaluate the quality of non-randomized studies. It is composed of 12 questions that focus on key concepts for evaluating the internal validity of a study. The assessment of the study heterogeneity was expressed by the Higgins $\mathrm{I}^{2}$ index (0-30\% low heterogeneity, $30-75 \%$ moderate heterogeneity and $75-100 \%$ high heterogeneity). A X2 based Q test was also performed to check between-study heterogeneity considering the cut-off for significance at $P<0.10$. Egger's regression test [18] was used to estimate the asymmetry of data and to elaborate Funnel Plots. The estimation of the Random effect was done by through the tausquared (т2).

Statistical analysis was conducted with Comprehensive Meta-Analysis Software (Version 3.3.070 - November 21, 2014), also used to develop the Forest Plots. Review Manager Software (Revman; The Cochrane Collaboration, Oxford, United Kingdom; version 5.3 - June 2014) was used to elaborate the Funnel Plots.

\section{Results}

\section{Study selection}

A total of 4,872 potentially relevant citations were identified. References were exported to Endnote for duplicates removal and were subsequently screened for relevance according to predefined inclusion and exclusion criteria. A total of 1,433 duplicate citations were removed and 3,209 studies were not included because they were clearly not in line with the meta-analysis eligibility criteria. Two interesting randomized LSG studies were excluded due to excessive mean baseline BMI $[19,20]$ One observational ESG study was not selected because of its inclusion criteria [21] One captivating manuscript on ESG was not originally written in English, so it was not included in our collection of studies [22]. Two studies met our inclusion criteria but had too short follow-up time [23, 24]. Two studies were excluded due to too small sample size $[25,26]$. Many that met our inclusion criteria did not provide data on 12 months follow up EWL\%, so their authors were contacted to get more detailed information; for some of them, we were kindly given a comprehensive answer [27-30]

One randomized study [31] and seven observational studies [27, $32-37]$ on LSG were eventually selected. Likewise, a total of eight observational studies [28-30,38-42] on ESG were included in the analysis ( $>$ Fig. 1 , $>$ Table 1 ).

\section{Patient characteristics}

As regarding the analysis of efficacy, the statistical analysis was conducted considering only groups of patients with a mean baseline BMI between 30 and $40 \mathrm{~kg} / \mathrm{m}^{2}$ who had completed 12 months follow-up after the procedure. Two Studies $[33,40]$ stratified their patient sample for classes of BMI; only class 1 (BMI between $30 \mathrm{~kg} / \mathrm{m}^{2}$ and $35 \mathrm{~kg} / \mathrm{m}^{2}$ ) and class 2 (BMI between $35 \mathrm{~kg} / \mathrm{m}^{2}$ and $40 \mathrm{~kg} / \mathrm{m}^{2}$ ) were included in our study sample. Relative to the analysis of safety, the statistical analysis was con-

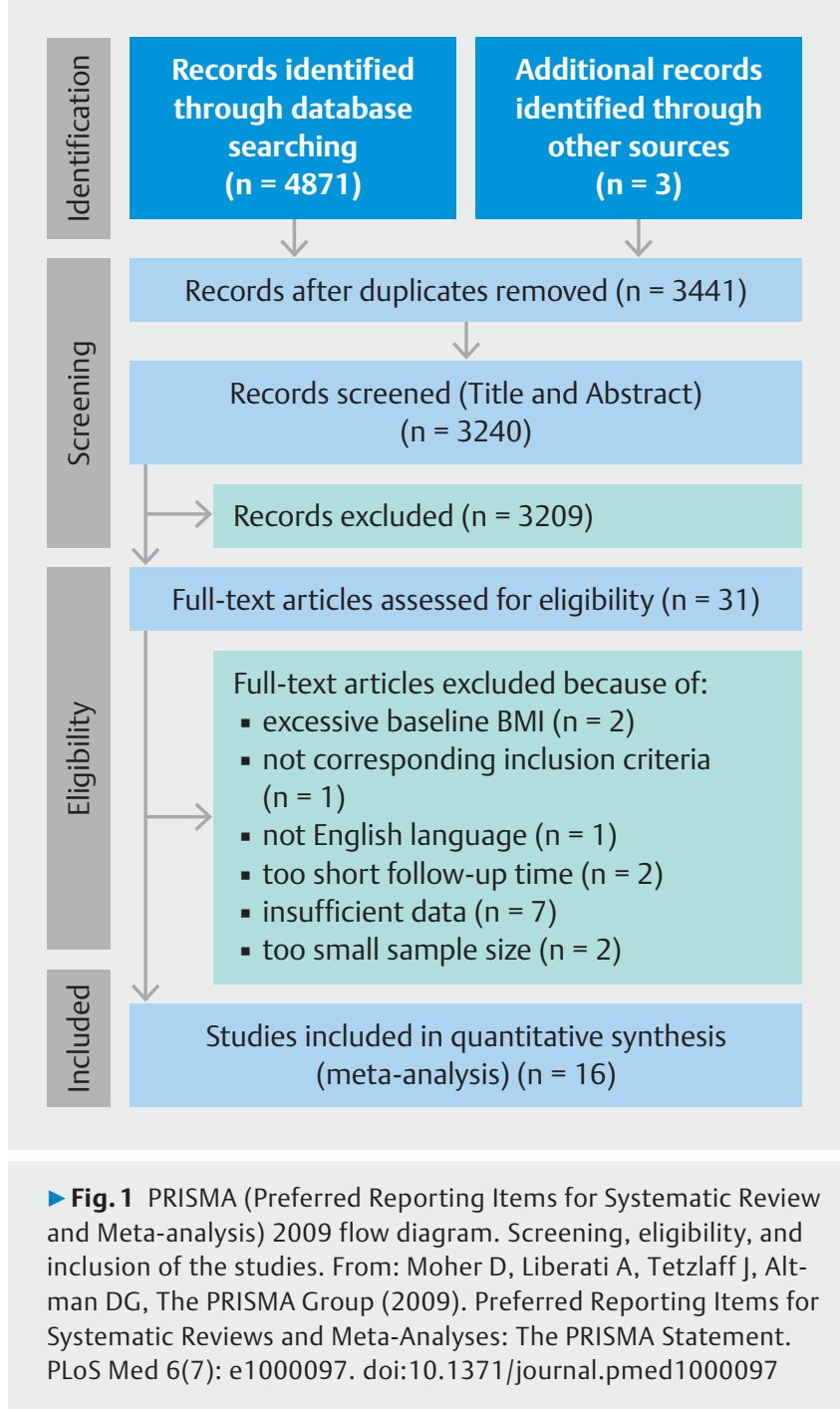

ducted considering all patients involved in the 16 studies included.

Overall, a total of 2188 participants were included in the analysis of the efficacy, defined as 12-month \%EWL; 1429 of them underwent LSG and 759 underwent ESG. A total of 3707 patients were included in the analysis of safety; 1929 of them underwent LSG and 1778 of them underwent ESG.

Mean age was $35.51 \pm 10.03$ years for the LSG group and $38.51 \pm 9.81$ years for the ESG group, with a mean difference of 3.00 years $( \pm 0,64 \mathrm{Cl}$; SE $0.326 P=0.0001) .79 .6 \%$ of the total population included were female. Mean preoperative BMI was $34.34 \pm 3.36 \mathrm{~kg} / \mathrm{m}^{2}$ for the LSG group and $34.72 \pm 4.73 \mathrm{~kg} / \mathrm{m}^{2}$ for the ESG group with a difference of $0.38 \mathrm{~kg} / \mathrm{m}^{2}( \pm 0.26 ; 95 \%$ $\mathrm{Cl} ; P=0.0046)$.

\section{Results of efficacy}

The details of our overall pooled mean \%EWL at 12 months in the LSG group (1429 patients) are shown in > Fig. 2a. The pooled mean \%EWL was $80.32 \%( \pm 12.20$; $95 \% \mathrm{Cl}$; Cochran's Q test $P=0.001)$, with a high grade of heterogeneity $\left(I^{2}=98.88\right.$, $\mathrm{T} 2=56.62)$. 


\section{衰管}

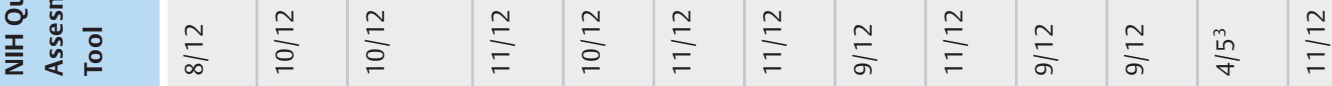

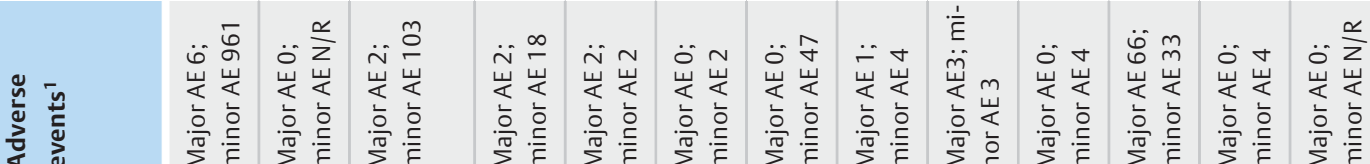

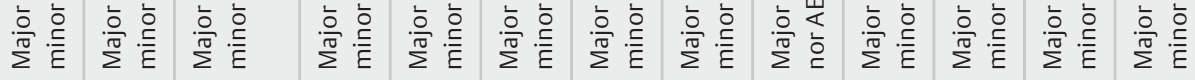

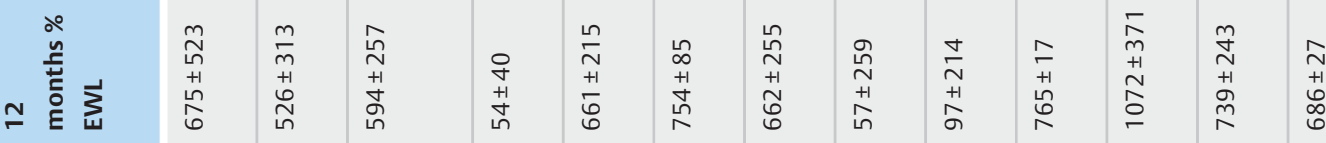

\begin{tabular}{|c|c|c|c|c|c|c|c|c|c|c|c|c|}
\hline 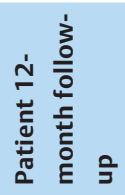 & $\stackrel{b}{\sim}$ & t & $\stackrel{\simeq}{\simeq}$ & $\stackrel{\sim}{\sim}$ & $\stackrel{8}{\circ}$ & $\stackrel{\infty}{ \pm}$ & $\mathscr{f}$ & $\stackrel{\vartheta}{q}$ & $\stackrel{\sigma}{\sim}$ & $\stackrel{N}{\simeq}$ & 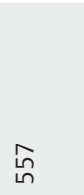 & $\stackrel{\mathscr{N}}{ }$ \\
\hline & $\begin{array}{l}m \\
\hat{0} \\
\dot{\Sigma}\end{array}$ & $\sum_{\Sigma^{0}}^{0} \stackrel{0}{L}^{\infty}$ & 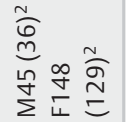 & $\frac{\bar{N}}{\sum^{\frac{N}{2}}}$ & $\begin{array}{l}0 \\
\infty \\
\sqcup \\
\dot{J} \\
\dot{\Sigma} \\
\Sigma\end{array}$ & $\begin{array}{l}\bar{N} \\
\underline{\Sigma} \\
\stackrel{\Sigma}{\Sigma} \\
\Sigma\end{array}$ & $\begin{array}{l}\stackrel{m}{+} \\
\stackrel{+}{0} \\
\dot{\Sigma}\end{array}$ & $\begin{array}{l}\frac{1}{N} \\
\dot{L} \\
0 \\
\dot{m} \\
\Sigma\end{array}$ & $\begin{array}{l}\infty \\
\infty \\
\\
\\
\\
\Sigma\end{array}$ & $\begin{array}{l}\frac{2}{\sigma} \\
\Sigma \\
\Sigma \\
\Sigma \\
\Sigma\end{array}$ & $\stackrel{\overrightarrow{0}}{\sum_{\Sigma}} \underset{i}{\mathbb{N}}$ & $\begin{array}{l}\stackrel{0}{N} \\
\stackrel{N}{\Sigma} \\
\Sigma\end{array}$ \\
\hline
\end{tabular}

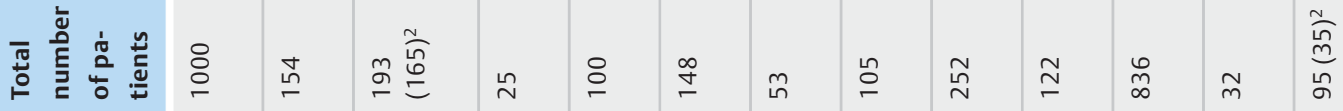

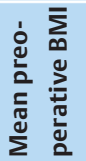

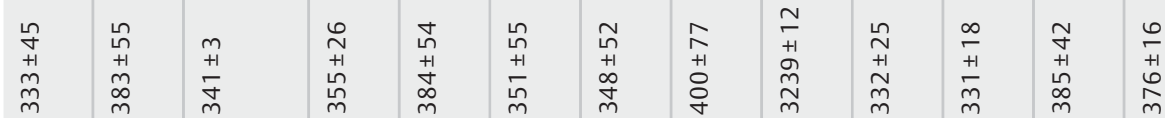

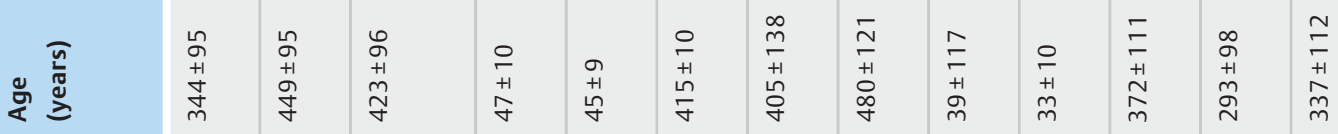

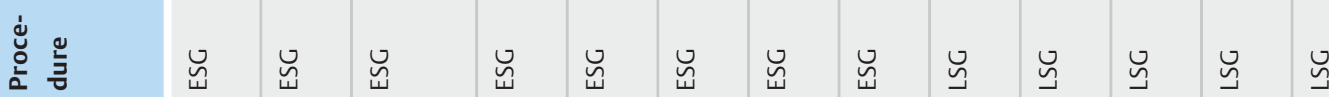

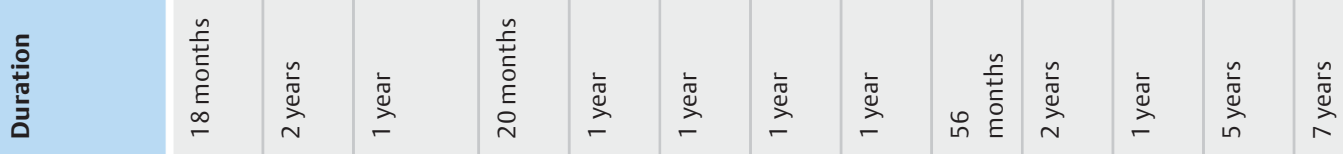

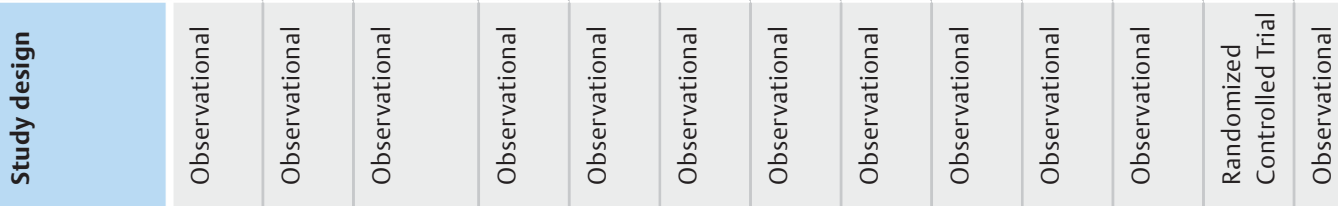

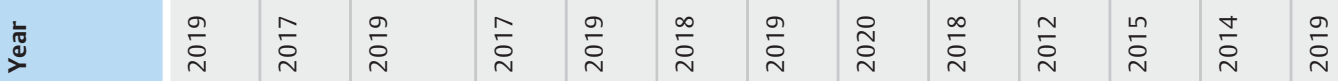

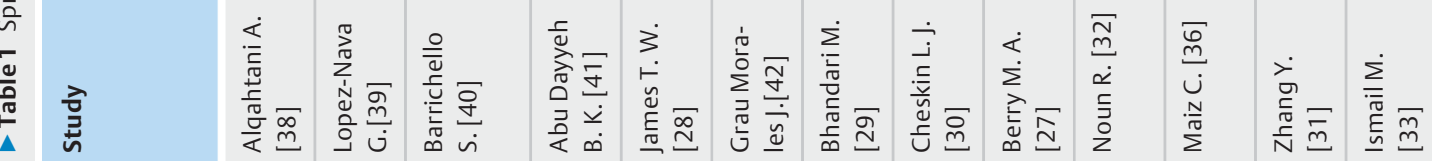




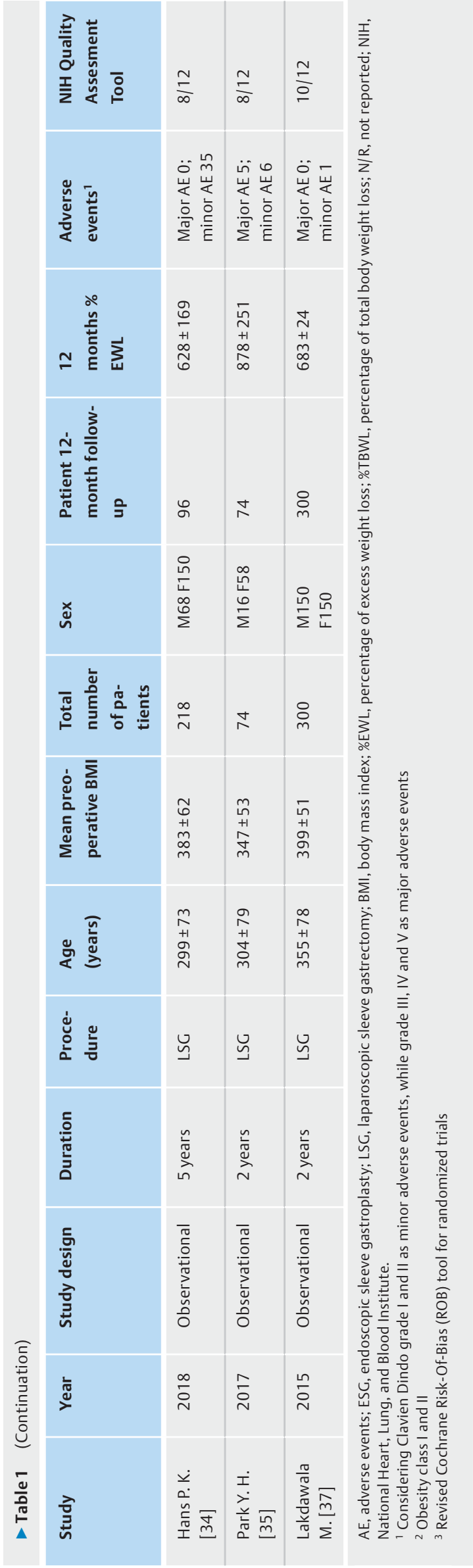

The details of our overall pooled mean \%EWL at 12 months in the ESG group (759 patients) are shown in $\mathbf{F i g . 2 b}$. The pooled mean \%EWL was 62.20\% $( \pm 4.38$; $95 \% \mathrm{Cl}$; Cochran's Q test $P=$ $0.005)$, with a moderate grade of heterogeneity $\left(I^{2}=65.52, \mathrm{~T} 2=\right.$ 24.68).

The difference in terms of \%EWL between the two samples was $18.12 \%( \pm 0.89 ; 95 \% \mathrm{Cl} ; P=0.0001)$.

\section{Results of safety}

The details of our overall pooled mean rate of major and/or minor AEs for the LSG group (1929 patients) are shown in > Fig. 3a. The pooled mean peri-procedural complications rate was $0.30 \%$ ( \pm 0.16 ; $95 \% \mathrm{Cl}$; Cochran's Q test $P=0.0001$ ), with a moderate grade of heterogeneity $\left(1^{2}=62.26\right)$.

The details of our overall pooled mean rate of major and/or minor AEs for the ESG group (1778 patients) are shown in - Fig. 3b. The pooled mean peri-procedural complications rate was $0.15 \%( \pm 0.07$; $95 \% \mathrm{Cl}$; Cochran's $Q$ test $P=0.0001)$ with a moderate grade of heterogeneity $\left(I^{2}=42.81\right)$.

The difference in terms of mean rate of major and/or minor AEs was $0.19 \%\left( \pm 0.37 ; 95 \% \mathrm{Cl} ; X^{2}=1.602\right)$ and it was not statistically significant $(P=0.2056)$.

\section{Risk of bias, study quality and heterogeneity}

The ROB tool ${ }^{13}$ and the NIH Quality Assessment Tool ${ }^{14}$ were used to assess the risk of bias for the randomized trials and the observational studies, respectively. The majority of the studies included revealed a moderate-to-high risk of bias, which translates to a rating of fair-to-poor quality of the studies. The main biases found in the selected studies were inevitably associated with the type of design: the people assessing the outcomes in all study considered were not blinded to the participants' interventions; eligibility/selection criteria for the study population were not often prespecified nor clearly described [28, 30, 34, $35,37]$; the participants in the study were frequently not representative of those who would be eligible for the intervention in the clinical population of interest [32-36]; sometimes the loss to follow-up rate was not reported or it was more than $20 \%$ [34-36, 38-40]; occasionally, outcome measures of interest were not taken multiple times before and after the intervention and statistical tests didn't provide $P$ values for the pre-to-post changes $[32,36,38]$. One study did not respect its own selection criteria, with a reported average BMI that was lower than the minimum value set [38].

With reference to the Clavien-Dindo classification [15], which deals with the stratification of peri-procedural complications, two studies did not report the rate of minor complications [33, 39]. Despite not being analyzed, further complications may occasionally have occurred.

Egger's regression test [18] used to estimate the asymmetry of 12 months \%EWL data among the enrolled articles about LSG and ESG led to the processing funnel plots shown in $>$ Fig. 4 and - Fig. 5, respectively. Both the LSG and ESG plots are asymmetrical, visually confirming the moderate-to-high grade of heterogeneity expressed by the Higgins $I^{2}$ index. Heterogeneity was higher among the LSG studies. 


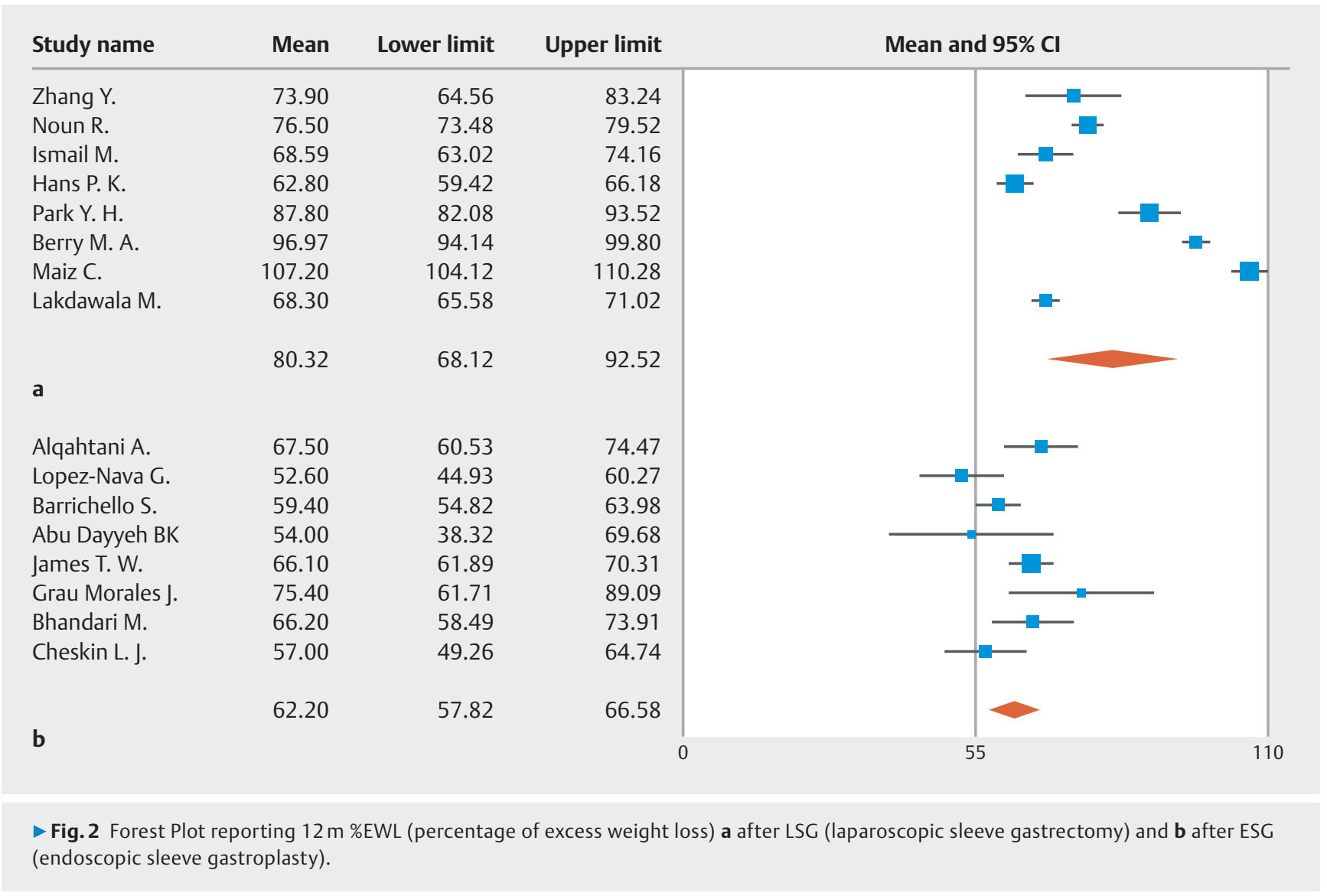

\section{Discussion}

LSG is the most popular bariatric surgery, providing effective weight loss and comorbidity improvement. With the progress of technology, less invasive endoscopic alternatives such as ESG have been proposed, whose initial purpose is to obtain the same results in terms of efficacy together with fewer complications. ESG is mainly proposed for patients with mild-to-moderate obesity, but there are still no guidelines that specify its applicability criteria. To compare for the first time the efficacy and safety of the endoscopic technique with the surgical one, we selected a cohort of patients affected by class I and class II obesity (BMI between 30 and $40 \mathrm{~kg} / \mathrm{m}^{2}$ ). Our result shows a statistically significant modest superiority for LSG compared with ESG in terms of excess weight loss after 12 months from the bariatric intervention. This can be attributed to the different mechanism of action of the two interventions. The surgical procedure irreversibly removes part of the gastric wall and, despite the elasticity of the remaining wall, it irreparably reduces the gastric volume. The endoscopic intervention, in contrast, does not deprive the gastric wall of any of its parts and provides the possibility of a suture failure, especially in conjunction with inadequate eating behavior of the patient. According to the results obtained, the incidence rate for AEs is not statistically different between the two groups. LSG leads to superior weight loss outcomes at 12 months with a similarly low AE rate compared with ESG.
The level of experience that has been achieved over time with regard to the surgical technique is, however, incomparable to the lack of confidence that we still have today with the endoscopic technique. This implies that a standardization of the endoscopic suture pattern and further refinements of the technology can lead to more satisfying results, both in terms of efficacy and safety.

The quality of the studies for which results were obtained often was poor. The design of most of the studies, in fact, revealed a moderate-to-high risk of bias. It appears that LSG is often proposed as a therapeutic approach for patients with class I obesity without comorbidities, not according to what is suggested by guidelines. The loss-to-follow-up rate after baseline is frequently high, especially in the ESG group, suggesting an inherent bias when analyzing the procedure outcomes. Furthermore, rates of minor AEs were not available for every study included; the lack of information can lead to miscalculated results.

The high grade of heterogeneity among the studies, which is more evident in the LSG group, testifies to the need for highquality double-blind randomized trials with adequate followup and post-procedural information, further standardization of the techniques, and specific guidelines to achieve uniformity in the surgery.

Based on our experience and on scientific evidence, we are facing a scenario in which ESG and LSG can be interpreted as therapeutic strategies applicable to different subclasses of ob- 


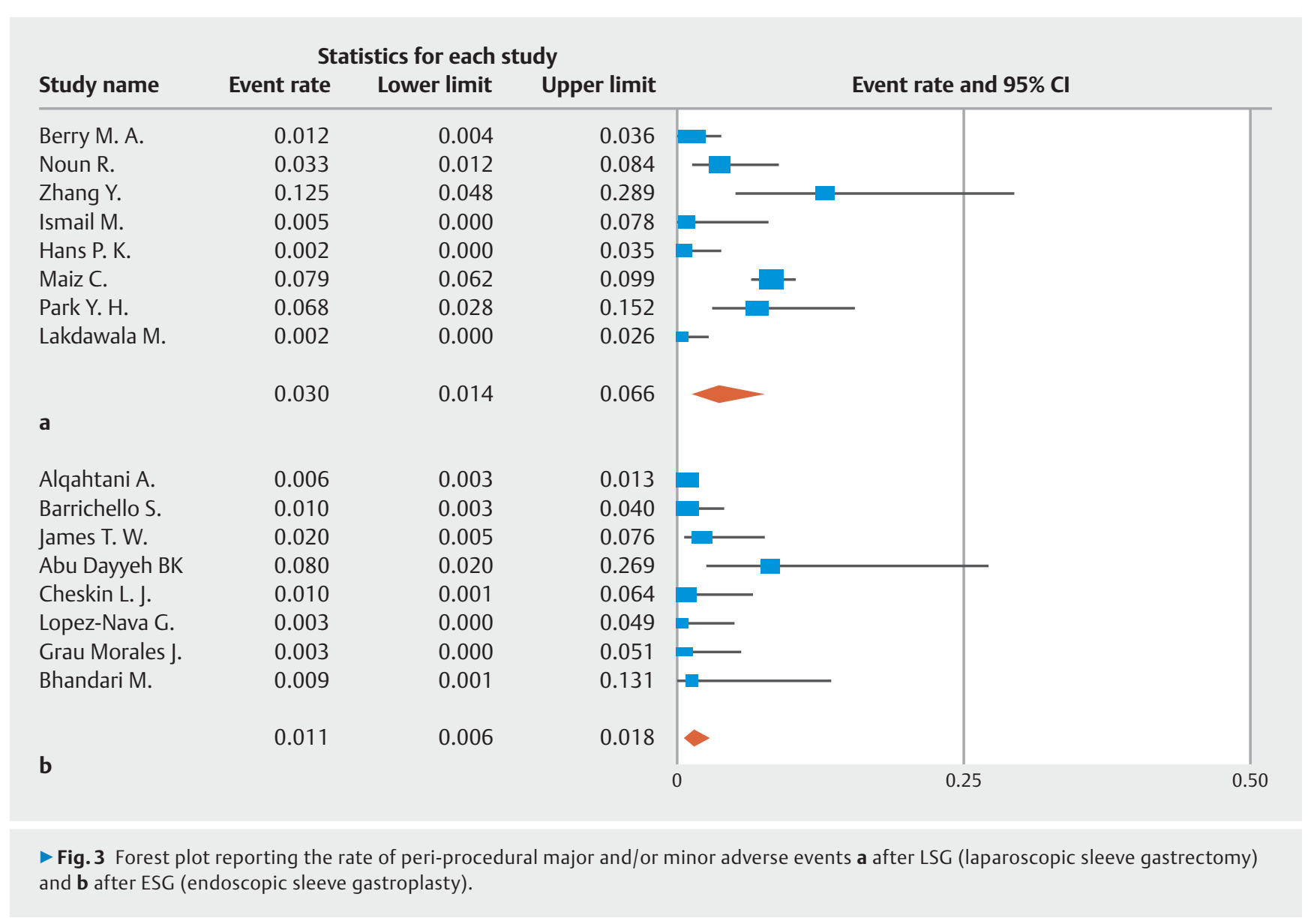

ese populations. LSG can be thought of as a treatment best suited for moderate-to-severe obesity and ESG as a therapeutic option for mild-to-moderate obesity. This provocative proposal could gain scientific validity if the correlation between the stratification of patients based on baseline BMI and the \%EWL obtained after ESG was demonstrated. It is possible, in fact, that patients subjected to ESG with lower baseline BMI have better outcomes in terms of \%EWL compared with patients with higher baseline BMI. In this scientific panorama, ESG could be considered not as a less-invasive and cost-effective alternative to LSG, but rather, as a procedure that improves the metabolic condition in patients suffering from mild-moderate obesity who did not get satisfying results through conservative therapeutic alternatives.

The multidisciplinary assessment of each patient facing any bariatric treatment option is mandatory.

\section{Conclusions}

In patients affected by mild or moderate obesity, \%EWL reported 12 months after LSG is moderately higher than that measured after ESG. The incidence of complications is not significatively different after the two procedures. The quality of the studies available is poor and further valid studies on which to base guidelines and, consequently, choices in daily clinical practice are an unmet need. We propose that surgical and endoscopic bariatric approaches should be considered as interventions whose therapeutic targets might be represented by distinct obese subpopulations.

\section{Competing interests}

Professor Guido Costamagna is consultant for Cook Medical, Boston Scientifc and Olympus. Dr. Ivo Bošskoski is consultant for Apollo Endosurgery, Cook Medical, Boston Scientific and Endo Tools, and is Apollo Endosurgery Research Grant Holder. 


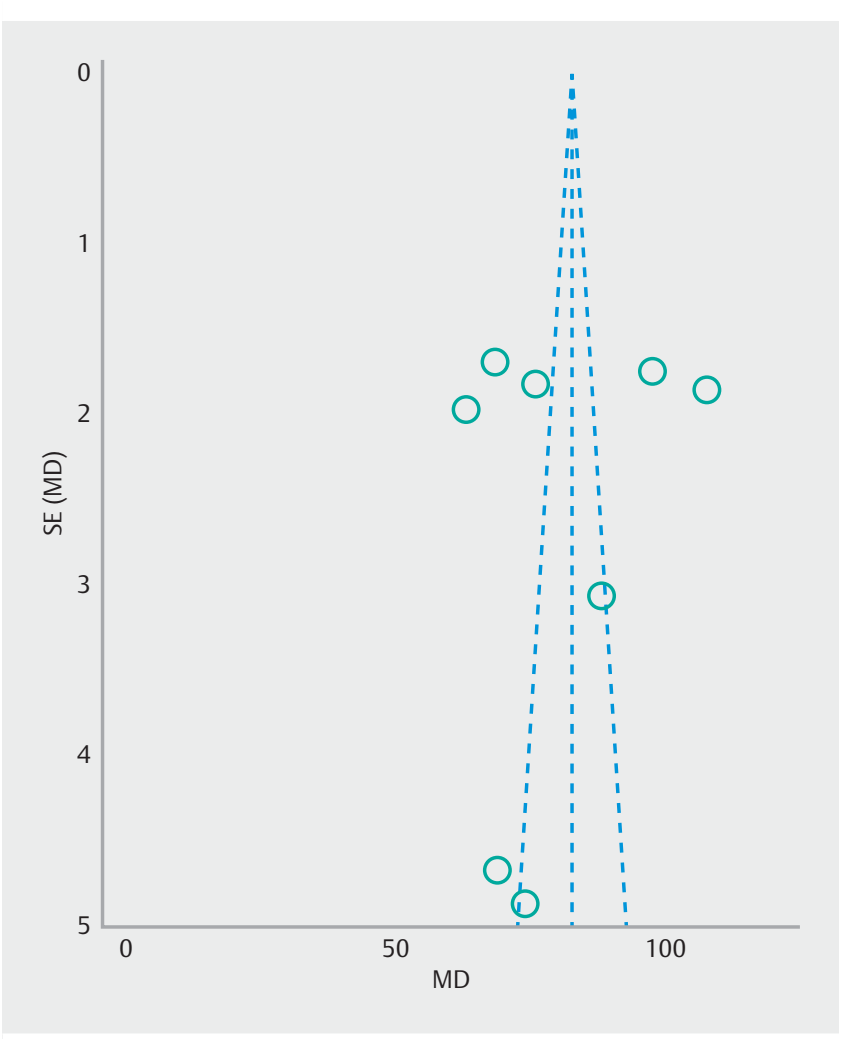

- Fig. 4 Funnel plot evaluating the potential publication bias among the enrolled articles LSC (laparoscopic sleeve gastrectomy).

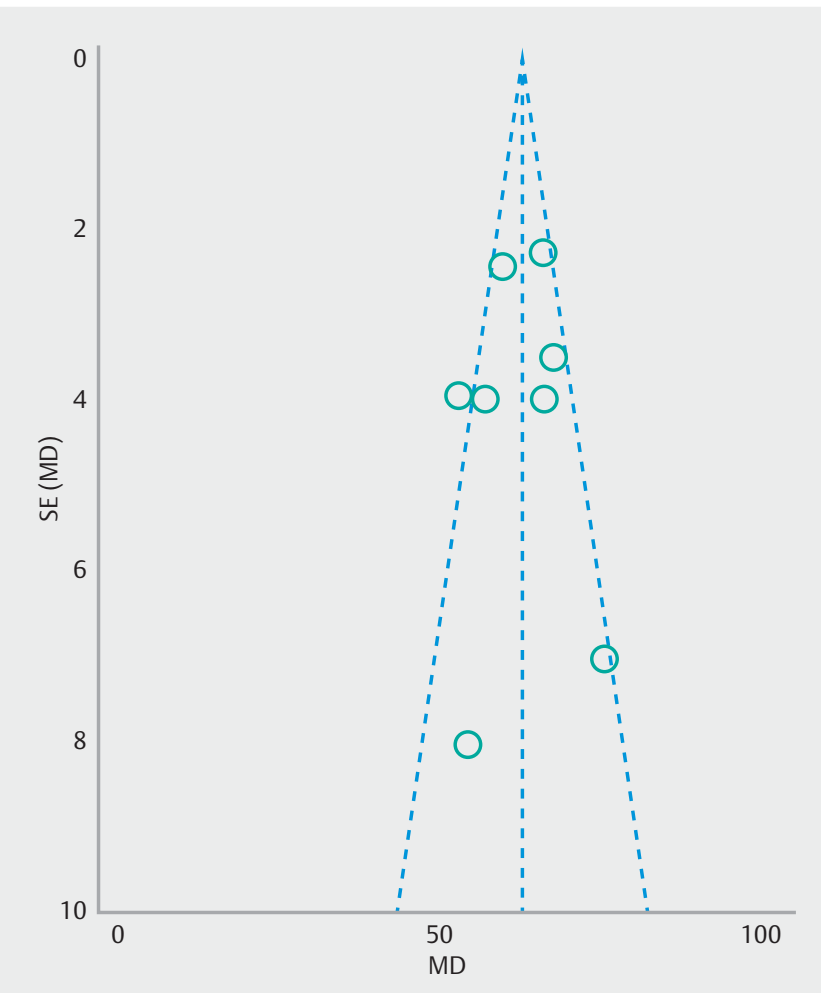

- Fig. 5 Funnel plot evaluating the potential publication bias among the enrolled articles about ESG (endoscopic sleeve gastroplasty).

\section{References}

[1] World Health Organization. Obesity and overweight. Available at (Accessed December 6, 2019): https://www.who.int/news-room/ fact-sheets/detail/obesity-and-overweight

[2] ASGE/ASMBS Task Force on Endoscopic Bariatric Therapy. A pathway to endoscopic bariatric therapies. Surg Obes Relat Dis 2011; 7: 672682

[3] American Society for Metabolic and Bariatric Surgery. Estimate of Bariatric Surgery Numbers 2011-2018. 26.06 2018: Available at (Accessed December 6, 2019): https://asmbs.org/resources/estimateof-bariatric-surgery-numbers

[4] Kheirvari M, Dadkhah Nikroo $\mathrm{N}$ et al. The advantages and disadvantages of sleeve gastrectomy; clinical laboratory to bedside review. Heliyon 2020; 6: e03496

[5] Boškoski I, Pontecorvi V, Gallo C et al. Redo endoscopic sleeve gastroplasty: technical aspects and short-term outcomes. Ther Adv Gastroenterol 2020; 13: 1756284819896179

[6] Storm AC, Abu Dayyeh BK. Endoscopic sleeve gastroplasty for obesity: defining the risk and reward after more than 1600 procedures. Gastrointest Endosc 2019; 89: 1139-1140

[7] The Cochrane Collaboration. 6.4.11.1 The Cochrane Highly Sensitive Search Strategies in MEDLINE. Available at (Accessed December 12, 2019): https://handbook-5-1.cochrane.org/chapter_6/ 6_4_11_1_the_cochrane_highly_sensitive_search_strategies_for. htm

[8] Moher D, Liberati A, Tetzlaff J et al. Preferred Reporting Items for Systematic Reviews and Meta-Analyses: The PRISMA Statement. PLOS Med 2009; 6: e1000097

[9] The Cochrane Collaboration. New Cochrane Handbook for Systematic Reviews of Interventions. Available at (Accessed December 12, 2019): news/new-cochrane-handbook-systematic-reviews-interventions

[10] The Cochrane Collaboration. Data extraction forms. Available at (Accessed December 12, 2019): https://dplp.cochrane.org/data-extraction-forms

[11] DerSimonian R, Laird N. Meta-analysis in clinical trials. Control Clin Trials 1986; 7: 177-188. doi:10.1016/0197-2456(86)90046-2

[12] Altman DG. Practical Statistics for Medical Research. Boca Raton, Fla: Chapman \& Hall/CRC; 1999

[13] Campbell I. Chi-squared and Fisher-Irwin tests of two-by-two tables with small sample recommendations. Stat Med 2007; 26: 3661-3675

[14] Richardson JT. The analysis of $2 \times 2$ contingency tables-yet again. Stat Med 2011; 30: 890-892

[15] Bolliger M, Kroehnert J-A, Molineus F et al. Experiences with the standardized classification of surgical complications (Clavien-Dindo) in general surgery patients. Eur Surg 2018; 50: 256-261

[16] The Cochrane Collaboration. RoB 2: A revised Cochrane risk-of-bias tool for randomized trials. Available at (Accessed December 6, 2019): bias/resources/rob-2-revised-cochrane-risk-bias-tool-randomizedtrials

[17] National Heart, Lung, and Blood Institute. Study Quality Assessment Tools. Available at (Accessed December 6, 2019): https://www.nhlbi. nih.gov/health-topics/study-quality-assessment-tools

[18] Egger M, Davey Smith G, Schneider M et al. Bias in meta-analysis detected by a simple, graphical test. BM] 1997; 315: 629-634

[19] Peterli R, Wölnerhanssen BK, Peters T et al. Effect of laparoscopic sleeve gastrectomy vs laparoscopic Roux-En-Y gastric bypass on weight loss in patients with morbid obesity: the SM-BOSS randomized clinical trial. JAMA 2018; 319: 255-265

[20] Kehagias I, Karamanakos SN, Argentou M et al. Randomized clinical trial of laparoscopic Roux-en-Y gastric bypass versus laparoscopic 
sleeve gastrectomy for the management of patients with $\mathrm{BMI}<50 \mathrm{~kg} /$ m2. Obes Surg 2011; 21: 1650-1656

[21] Saumoy M, Schneider Y, Zhou XK et al. A single-operator learning curve analysis for the endoscopic sleeve gastroplasty. Gastrointest Endosc 2018; 87: 442-447

[22] Espinet Coll E, López-Nava Breviere G, Nebreda Durán J et al. Spanish consensus document on bariatric endoscopy part 1. general considerations. . Rev Espanola Enfermedades Dig 2018; 110: 386-399

[23] Sartoretto A, Sui Z, Hill C et al. Endoscopic sleeve gastroplasty (ESG) is a reproducible and effective endoscopic bariatric therapy suitable for widespread clinical adoption: a large, international multicenter study. Obes Surg 2018; 28: 1812-1821

[24] Fayad L, Adam A, Schweitzer M et al. Endoscopic sleeve gastroplasty versus laparoscopic sleeve gastrectomy: a case-matched study. Gastrointest Endosc 2019; 89: 782-788

[25] Lee W-J, Ser K-H, Chong K et al. Laparoscopic sleeve gastrectomy for diabetes treatment in nonmorbidly obese patients: efficacy and change of insulin secretion. Surgery 2010; 147: 664-669

[26] Shen D, Ye H, Wang Y et al. Comparison of short-term outcomes between laparoscopic greater curvature plication and laparoscopic sleeve gastrectomy. Surg Endosc 2013; 27: 2768-2774

[27] Berry MA, Urrutia L, Lamoza P et al. Sleeve gastrectomy outcomes in patients with BMI between 30 and 35-3 years of follow-Up. Obes Surg 2018; 28: 649-655

[28] James TW, McGowan CE. The descending gastric fundus in endoscopic sleeve gastroplasty: implications for procedural technique and adverse events. VideoGIE Off Video 2019; 4: 254-255

[29] Bhandari M, Jain S, Mathur W et al. Endoscopic sleeve gastroplasty is an effective and safe minimally invasive approach for treatment of obesity: First Indian experience. Dig Endosc 2019: doi:10.1111/ den.13508

[30] Cheskin L], Hill C, Adam A et al. Endoscopic sleeve gastroplasty versus high-intensity diet and lifestyle therapy: a case-matched study. Gastrointest Endosc 2020; 91: 342-349.e1

[31] Zhang Y, Zhao H, Cao Z et al. A randomized clinical trial of laparoscopic Roux-en-Y gastric bypass and sleeve gastrectomy for the treatment of morbid obesity in China: a 5-year outcome. Obes Surg 2014; $24: 1617-1624$
[32] Noun R, Chakhtoura G, Nasr M et al. Laparoscopic sleeve gastrectomy for mildly obese patients (body mass index of $30<35 \mathrm{~kg} / \mathrm{m} 2$ ): operative outcome and short-term results. . J Obes 2012; 2012: 813650

[33] Ismail M, Nagaraj D, Rajagopal M et al. Seven-year outcomes of laparoscopic sleeve gastectomy in Indian patients with different classes of obesity. Obes Surg 2019; 29: 191-196

[34] Hans PK, Guan W, Lin S et al. Long-term outcome of laparoscopic sleeve gastrectomy from a single center in mainland China. Asian J Surg 2018; 41: 285-290

[35] Park YH, Kim SM. Short-term outcomes of laparoscopic greater curvature plication and laparoscopic sleeve gastrectomy in patients with a body mass index of 30 to $35 \mathrm{~kg} / \mathrm{m} 2$. Yonsei Med J 2017; 58: 10251030

[36] Maiz C, Alvarado J, Quezada N et al. Bariatric surgery in 1119 patients with preoperative body mass index $<35 \mathrm{~kg} / \mathrm{m} 2$ : results at 1 year. Surg Obes Relat Dis 2015; 11: 1127-1132

[37] Lakdawala M, Agarwal A, Dhar S et al. Single-incision sleeve gastrectomy versus laparoscopic sleeve gastrectomy. A 2-year comparative analysis of 600 patients. . Obes Surg 2015; 25: 607-614

[38] Alqahtani A, Al-Darwish A, Mahmoud AE et al. Short-term outcomes of endoscopic sleeve gastroplasty in 1000 consecutive patients. Gastrointest Endosc 2019; 89: 1132-1138

[39] Lopez-Nava G, Sharaiha RZ, Vargas EJ et al. Endoscopic sleeve gastroplasty for obesity: a multicenter study of 248 patients with 24 months follow-up. Obes Surg 2017; 27: 2649-2655

[40] Barrichello S, Hourneaux de Moura DT, Hourneaux de Moura EG et al. Endoscopic sleeve gastroplasty in the management of overweight and obesity: an international multicenter study. Gastrointest Endosc 2019; 90: 770-780

[41] Abu Dayyeh BK, Acosta A, Camilleri M et al. Endoscopic sleeve gastroplasty alters gastric physiology and induces loss of body weight in obese individuals. Clin Gastroenterol Hepatol Off Clin Pract 2017; 15: 37-43.e 1

[42] Graus Morales ], Crespo Pérez L, Marques A et al. Modified endoscopic gastroplasty for the treatment of obesity. Surg Endosc 2018; 32: 3936-3942

\section{CORRECTION}

Giuseppe Marincola, Camilla Gallo, Cesare Hassan et al. Laparoscopic sleeve gastrectomy versus endoscopic sleevegastroplasty: a systematic review and meta-analysis

Endoscopy International Open 2021; 09: E87-95.

DOI: $10.1055 / a-1300-1085$

Giuseppe Marincola ${ }^{1}$, Camilla Gallo ${ }^{2,3}$, Cesare Hassan ${ }^{4}$,

Luca Sessa ${ }^{1}$, Marco Raffaelli ${ }^{1}$, Guido Costamagna ${ }^{2,3}$, Vincenzo Bove ${ }^{2,3}$, Valerio Pontecorvi ${ }^{2,3}$, Beatrice Orlandini ${ }^{2,3}$, Ivo Boškoski,

Institutions

${ }^{1}$ Bariatric and Metabolic Surgery Unit, Fondazione Policlinico Universitario Agostino Gemelli IRCCS, Rome, Italy

2 Digestive Endoscopy Unit, Fondazione Policlinico Universitario Agostino Gemelli IRCCS, Rome, Italy

${ }^{3}$ Centre for Endoscopic Research Therapeutics and Training (CERTT), Catholic University of Rome, Italy

${ }^{4}$ Digestive Endoscopy Unit, Nuovo Regina Margherita, Rome, Italy

The author Luca Sessa was missing. 